\title{
Ann Arbor Pathologic Stage III
}

National Cancer Institute

\section{Source}

National Cancer Institute. Ann Arbor Pathologic Stage III. NCI Thesaurus. Code

C125477.

A pathologic stage that refers to lymphoma involving node regions on both sides of the diaphragm, or an extralymphatic organ or site plus nodes on both sides of the diaphragm (IIIe), or lymph nodes on both sides of the diaphragm plus spleen (IIIs). 